Helaba: A System to Highlight Design Rationale in Collaborative Design Processes Peer-reviewed author version

GUTIERREZ LOPEZ, Marisela; HAESEN, Mieke; LUYTEN, Kris \& CONINX, Karin (2015) Helaba: A System to Highlight Design Rationale in Collaborative Design Processes. In: Proceedings of the 12th International Conference on Cooperative Design, Visualization, and Engineering.

Handle: http://hdl.handle.net/1942/19664 


\title{
Helaba: A System to Highlight Design Rationale in Collaborative Design Processes
}

\author{
Marisela Gutierrez Lopez, Mieke Haesen, Kris Luyten, Karin Coninx \\ Hasselt University - tUL - iMinds, Expertise Centre for Digital Media \\ Wetenschapspark 2, 3590 Diepenbeek, Belgium \\ \{marisela.gutierrezlopez, mieke.haesen, kris.luyten, \\ karin.coninx\} @uhasselt.be
}

\begin{abstract}
Design activities associated to the ideation phase of design processes require mutual understanding and clear communication based on artefacts. However, this is often a challenge for remote and multidisciplinary teams due to the lack of ad hoc tools for this purpose. Our approach is to solve these limitations by explicitly connecting pieces of information related to design rationale, feedback, and evolution with the artefacts that are subject of communication. We propose Helaba, a system that creates a shared workspace to support communication revolving around design artefacts and activities within multidisciplinary teams. Helaba supports design communication and rationale, and potentially leads to more satisfying outcomes from the design process.
\end{abstract}

Keywords: Design, design rationale, remote / multidisciplinary communication

\section{Introduction}

Modern design has evolved into a complex knowledge activity that more often than not involves multidisciplinary teams. However, these teams often lack the tools for efficient collaboration and communication, specifically because the different roles involved also have different work practices and focus, and use domain specific languages.

We tackle two main challenges in communication within a design team and with their stakeholders. First, issues with mutual understanding and clear communication during the ideation phase of the design process, which leads to suboptimal results, loss of time and thus money and sometimes frustrations and difficulties in collaboration. Second, design processes often involve asynchronous and remote communication, which we found has many disadvantages if there is no domain-specific system to mediate and support this type of communication. We discovered that explicitly connecting pieces of information with the artefacts that are the subject of communication can help a great deal to improve this.

In this paper we introduce Helaba, a semi-functional prototype that aims to reduce the challenges faced in remote, multidisciplinary design communication. The word Helaba is used in the Flemish spoken language to greet someone, but also to attract the

adfa, p. 1, 2011.

(C) Springer-Verlag Berlin Heidelberg 2011 
explicit attention of another person and point something out. The Helaba prototype focuses on the processes for documenting design rationale and decision-making since they have been acknowledged to support multidisciplinary communication. Section 2 of this paper elaborates on how our prototype relates to previous research.

The concept of Helaba, presented in section 3, is based on previous research where a survey and series of interviews uncovered that designers consider team and customer communication the main issue that influences the quality of their designs and design decisions, rather than technical limitations they might have to deal with [1]. Based on this study, we analysed and mapped internal and external (customer and other stakeholders) communication, both on the design activities and on the artefacts that are subject of these activities.

Rather than creating a system that integrates everything in one single tool (e.g. chat, e-mail), Helaba focuses on the visualization of communication and artefact evolution over time. Because of this focus, Helaba helps to structure, understand and optimize the team communication as well as the rationale that led to certain design decisions. Helaba does not include artefact editing or manipulation and leaves this work to specialized design tools. The focus of Helaba ensures that different disciplines involved in design teams can adopt this tool without moving away from the tools they are familiar with.

Sections 4 and 5 present the lessons learned from three scenarios about the way Helaba supports communication within multidisciplinary, distributed design teams. While this solution is an initial insight that needs further validation, the proposed prototype is a system for "smoothening" communication. It can be integrated into the design process, and is adoptable by both team members and stakeholders. Future steps towards this integration are discussed in section 6 .

\section{Context and Background}

Communication during the early stages of design is often linked to visual artefacts [2, 3]. However, existing systems tend to focus on the support for creating these artefacts, but not on the influence of communication to their evolution [3]. Designers use various practices as workarounds to reduce the burden of managing artefact-based communication, including [1]:

- Crafting textual communication to "point" at specific elements of artefacts. However, designers must invest a significant amount of resources in this task.

- Having multiple channels of feedback and communication. This strategy implies that designers frequently fail to capture ideas together with artefacts, as communication is scattered among different tools.

- Using physical workspaces to convey within multidisciplinary teams, organizing frequent meetings and workshops with stakeholders for reaching common ground. This is difficult in remote teams, as moments of face-to-face meetings are limited.

These practices are consistently mentioned as helpful but often frustrating and a common source of misunderstandings [1]. In this paper we propose a system to address this gap, reflecting on a solution to integrate artefacts and design decisions. 
A useful approach for integrating communication and artefacts is by capturing the design rationale. Design rationale is a representation of the reasoning behind the design of an artefact, evolving with the design process by capturing design decisions and how they relate to relevant evaluation criteria [4]. Explicitly documenting design rationale is useful for connecting discussions and communications into artefacts [5]. Furthermore, design rationale is useful when artefacts need to be understood by many people, allowing them to better comprehend the design decisions of others and keep track of previous group decisions [4].

Existing systems for capturing design rationale focus on a variety of methods and approaches. Some of these systems have focused on capturing design rationale based on traceability of requirements [6], argumentation [7], and decision-making processes [8]. While each of these systems propose a valuable approach for capturing design rationale, design practitioners are yet to adopt tools with this purpose [9]. One reason for this is that designers customize existing tools to their own practices [1]. Therefore, efforts should be directed to a solution that can be adapted to a variety of design practices. Helaba extends previous work by proposing a tool for capturing design rationale by connecting pieces of information related to rationale, feedback, and evolution of artefacts to the communication and design activities of multidisciplinary design teams.

Some characteristics of Helaba are inspired by Design Space Analysis (DSA) [4], which is an approach for representing design rationale in a simple and flexible way by opening a space for augmenting the rationale elements. According to [4], DSA uses a semi-formal notation called QOC, which stands for Question, Options, Criteria, to outline the design space of artefacts. The elements of the QOC notation are: Questions, which identify discussions related to the artefact, Options, which provide trade-offs and answers to the Questions, and Criteria, which define the evaluation criteria for an Option. Helaba is related to the DSA approach as we adopt the understanding of design rationale as: (i) an artefact that has to be crafted and evolved along the design process, and, (ii) a discussion space for ongoing issues [4]. Furthermore, Helaba's workspace was inspired by the QOC notation, as we represent argumentation of options in relation to its evaluation criteria.

\section{Helaba Design Overview}

Helaba is a system prototype that supports communication revolving around design artefacts and activities within multidisciplinary teams. A shared workspace that structures and tracks artefacts and design activities is the main component [10]. Three core User Experience (UX) guidelines were used as design rules for Helaba. These UX guidelines are the result from our user studies with design practitioners, which reported the importance of capturing, structuring, and presenting communication within design teams and with stakeholders [1]. The UX guideline 1 indicates that when design teams communicate, there should exist a connection between the tools for sharing artefacts and for communicating design rationale to facilitate the creation of a common visual vocabulary. UX guideline 2 points out that when designers share and receive feedback 
from their team or stakeholders, a shared, activity-oriented workspace should be integrated to trace back the design decisions and rationale behind them. Finally, UX guideline 3 specifies that when involved in artefact-based communication, designers need to maintain awareness over the evolution of the artefact, having an overview of previous design decisions.

In consideration of these three UX guidelines, our prototype is designed to focus on communication related to the design activities and artefacts. We believe that this will improve documenting the design rationale significantly, and eventually lead to more satisfying outcomes from the design process. Figure 1 is a screen shot of Helaba that demonstrates its features. The images of avatars and the sketches are examples of content created during the design process, which are used for illustrative and expert review purposes, but should not be considered as part of the prototype.

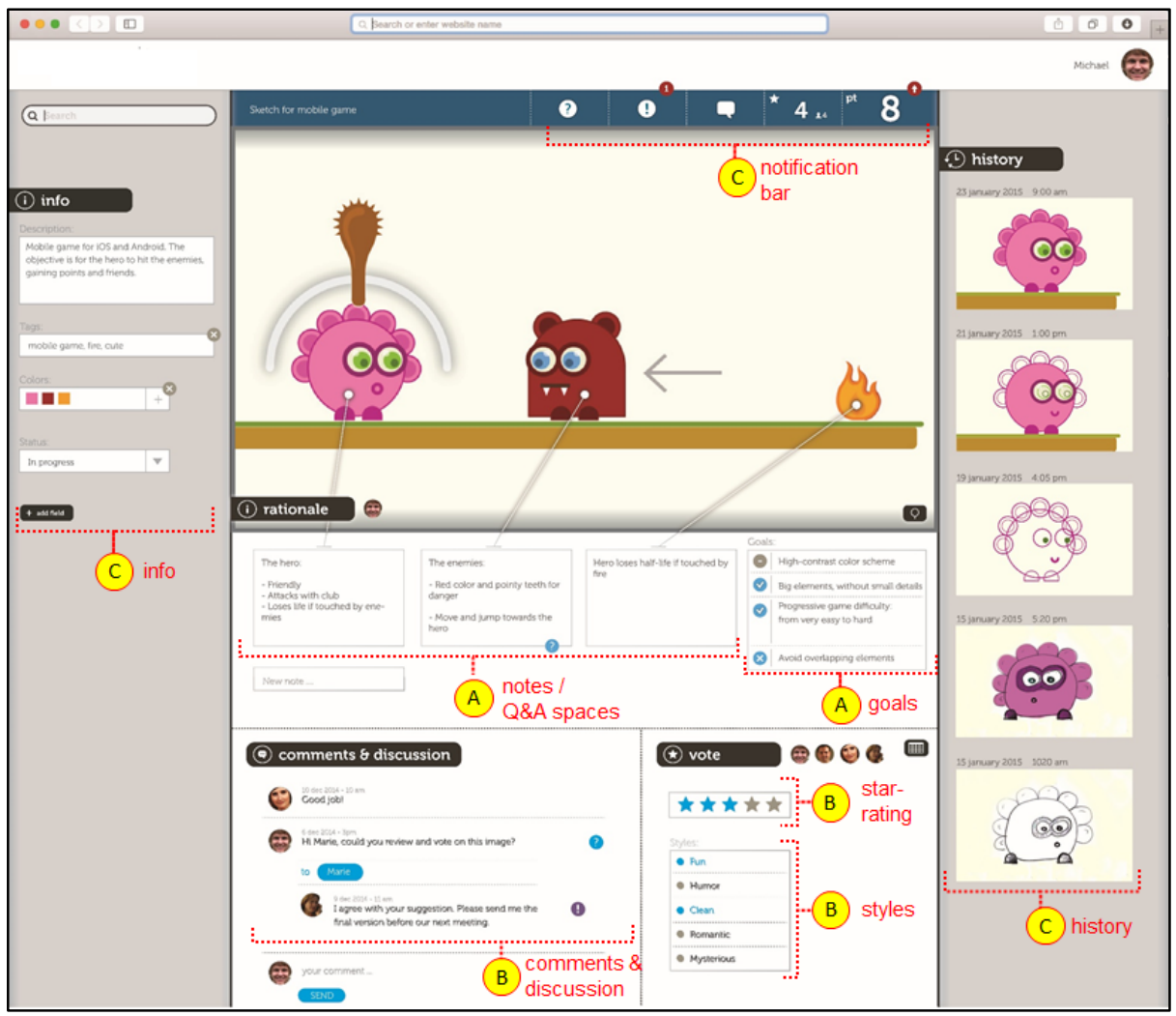

Fig. 1. Screenshot of Helaba labelling the (A) rationale, (B) feedback, and (C) evolution information types and their associated features.

The information captured and presented by Helaba can be decomposed in three different types of information, which each include a set of features and deal with a UX guideline. (i) The intention of the rationale information (Figure 1, A), which reflects 
on UX guideline 1, is to document the reasoning and arguments of the artefact in relation to its evaluation criteria, as suggested in the QOC notation. (ii) Feedback information (Figure 1, B) reflects on UX guideline 2 as it organizes general discussions and gathers qualitative and quantitative feedback. (iii) Evolution information (Figure 1, C) refers to UX guideline 3 and concerns the metadata of the artefact and timeline for traceability of its evolution.

\section{$4 \quad$ Making Design Decisions and Rationale Explicit}

For exploring potential uses of Helaba, we observed the ideation processes of a multidisciplinary team involved in a user interface (UI) design project. The two main reasons to observe this particular team and project are: (i) the multidisciplinary nature of UI design projects, and (ii) the involvement of complex design and technological problems being solved by geographically distributed team members. The observed UI design project involves 6 organizations from both industrial and academic fields distributed across different locations in Europe. The team, formed by 11 professionals, worked towards the common goal of creating a shared UI design for an interactive system.

The analysis of these observations led to the creation of three open-ended and fragmentary narrative scenarios that concentrate on the different communication activities associated to ideation processes. In the scenarios the design team consists of 4 key actors: Joe a UX designer, Pam a visual designer, Ann a software developer, and Danny the project manager. Each scenario presented below, which is based on our observations, describes a pitfall in the communications of the team. Following, a description of Helaba's features explain how Helaba can overcome the pitfall for each scenario.

\subsection{Documenting Outcomes of Individual Work}

Joe individually created early UI designs. He focused on designing UIs using Illustrator while making sketches and writing annotations in his notebook and on random pieces of paper. He worked with ease in these individual activities, but lost track of the reasoning behind the evolution of his designs since he had no centralized record of his ideas. This made difficult for Joe to accurately communicate his sources of inspiration and evolution of ideas to his team.

Joe fell in what we call the scattered rationale pitfall, as he does not have a centralized record of how his design came to be. This can create difficulties when transitioning from individual to group work. Helaba can be helpful for capturing the rationale information with two features (Figure 1, A). The notes feature supports digital "post-its" that can be used to include textual notes, and pinpoints to specific elements of the design. Individual notes can be transformed into $Q \& A$ spaces, which are open spaces for discussion, as designers can directly ask questions to specific team members (see Figure 2 ). This feature could be useful to integrate communication that currently takes place over e-mails or chat messages. The goals feature allows the creation of a customizable checklist containing design guidelines or requirements that serve as criteria for evaluation of the artefact. Criteria can be checked once they are handled in the artefact. 


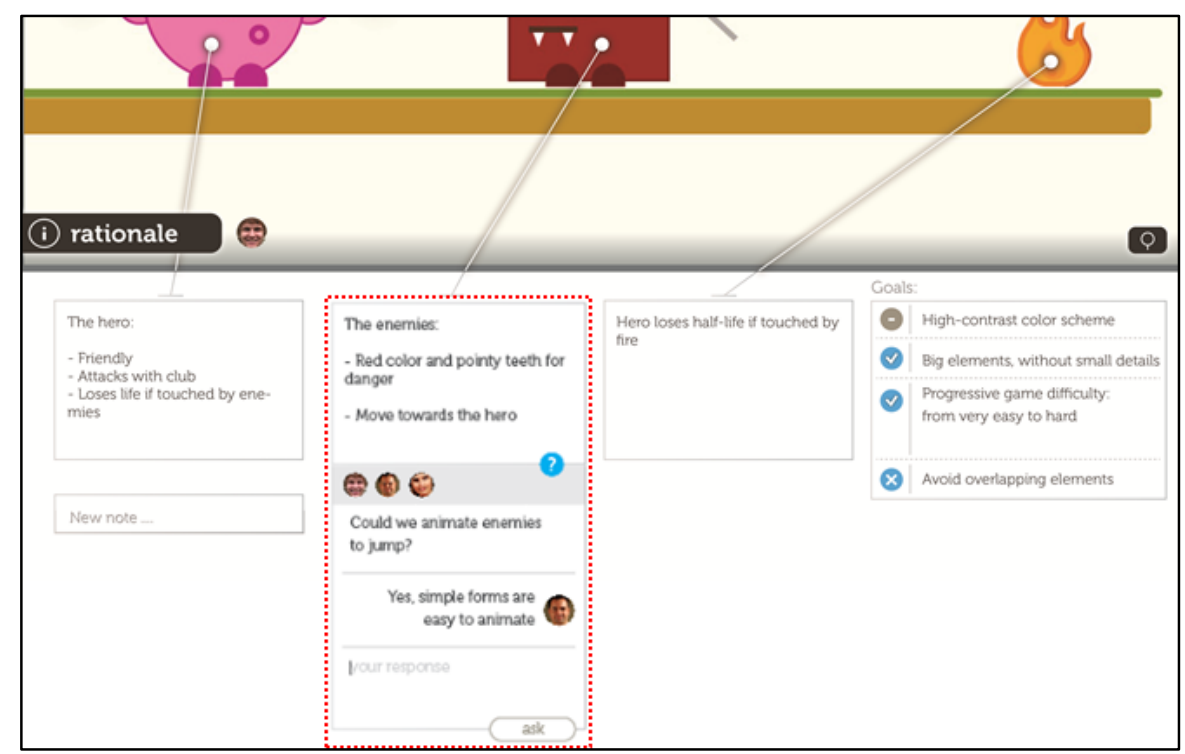

Fig. 2. Notes feature with active $Q \& A$ space and goals feature with checked items.

\subsection{Communicating Feedback within Multidisciplinary Teams}

Joe organized a VoIP call with screen sharing with Pam and Ann to gather feedback about his UI designs. During Joe's remote presentation, Ann felt it was not the place for asking questions about implementation, while Pam felt her ideas were not valuable enough to interrupt his speech. The outcomes of this meeting were poor for the participants. From Joe's perspective, the reactions of his UI design were positive because he gathered only a few lines of feedback. From Ann's and Pam's perspective, they were not able to express their points of view in full.

The team fell in what we call the incomplete feedback pitfall. This means that remote communication often leads to a loss of context of what actually happens. Helaba aims to overcome this pitfall by integrating and facilitating the capture of feedback information (Figure 1, B). The comments \& discussion feature is a space for global argumentation. Individual comments can be marked to direct an inquiry (question) or to bring awareness (attention) to a specific team member. The vote feature is a space to give feedback in a quick, visual way. Users can vote using a 5 points star-rating scale and a styles scale, which are user-defined categories that can be used to rate and interpret an artefact. This is illustrated in Figure 3, where five categories are defined by the designer and open for others to vote, giving designers the opportunity to evaluate the perceptions of their team about the artefact. 


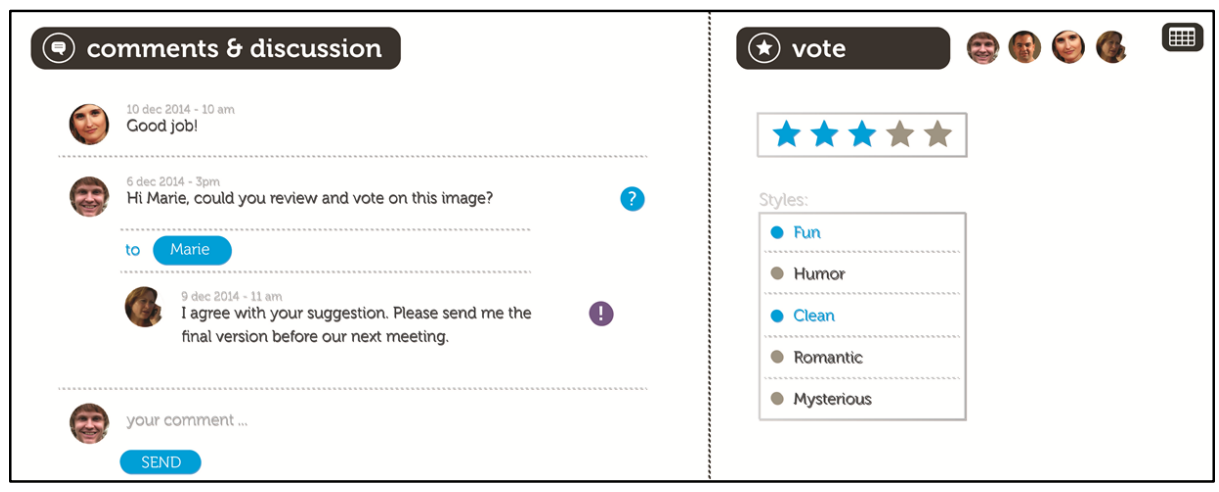

Fig. 3. Left side: comments \& discussion feature with comments marked as question or attention. Right side: vote feature with star-rating scale and styles suggestions.

\subsection{Communicating Design Decisions to Stakeholders}

Joe, Pam, Ann, and Danny gathered together for a co-located workshop. Since Danny was not involved in the iterative process for this artefact (scenario 2), he had several remarks about Joe's UI designs. Not having a clear focus, the team discussion started to circle around previously agreed design decisions. This was a critical point of the project, as it could lead to wasting valuable ideas or resources.

Danny fell in the multidisciplinary decision-makers pitfall. The evolution information kept by Helaba can be useful to overcome this pitfall, as Danny was not informed of how to proceed due to misinformation. The features of Helaba that can be useful are (Figure 1, C): history, a timeline to record the evolution of an artefact's elements in chronological order. The notification bar, that includes a general rating of image, which is a numeric grade ( 1 to 9 ) to qualify the acceptance rate of the image (i.e. star-rating) and the adherence to requirements (i.e. goals).

\section{Discussion}

Our approach focuses on supporting communication related with design activities and artefacts. Designers report that most of their collaborative problems are due to problematic communication instead of technical limitations [1]. Despite many different remote communication technologies that are available today, we found that existing solutions fail to support communication on design activities and artefacts. The three pitfalls described in the previous section highlight missing features of current communication tools for designers. Helaba fills this gap as it provides solutions for these pitfalls by structuring the communication following design rules. These rules were obtained from interviews with designers and observing collaborative design activities. To validate our approach, we conducted expert reviews with 8 design practitioners to explore the concept of Helaba. From this early validation with domain experts, we discovered 
opportunities for refining Helaba, but most importantly, that our approach has the potential to set the ground to create more comprehensive development of communication tools to support design activities. Besides enabling traceability of the design rationale and reflecting on team-based design practices, Helaba solves the pitfalls that were identified as the major causes of work dissatisfaction during our observations and interviews.

\subsection{Transitioning from Individual to Group Work with Helaba}

Designers invest much of their time and efforts working individually on their designs, but not on documenting the reasoning behind artefacts $[5,10]$. Nevertheless, when transitioning from individual to group work, designers must find ways to communicate the design rationale to their team members and stakeholders [5]. A common practice for communicating design rationale is to write the rationale, posting it into e-mails or Basecamp, and embedding the artefact. Designers mention this as inefficient, as they invest plenty of time and resources on crafting the rationale that is often misinterpreted since it is not attached directly to the artefact. This is the scattered rationale pitfall.

Helaba creates a centralized workspace to embed rationale information to artefacts by means of notes, comments, discussions and evaluation criteria, enabling the inclusion of informal communication. This information is captured along the design process and shared directly with team members. Currently this information is dispersed and often hard to connect to design artefacts as designers have no explicit support for storing and sharing it. Helaba could solve this issue as illustrated by a domain expert.

"[The notes feature is] ... very useful to connect to the part I'm talking about. Now I just type long emails, or a document with all comments, and they [team members] have to search what I am talking about. I would use it a lot." [Graphical designer]

\subsection{Integrating Multidisciplinary Points of View with Helaba}

In multidisciplinary teams, designers must consider feedback from a variety of perspectives $[3,4,5]$. Current practices for gathering feedback include using video conferencing and screen sharing tools for organizing remote, synchronous meetings. However, remote feedback implies a reduction of communication cues and a loss of human and work context (e.g. empathy [12]), which makes it difficult to assess the reactions of others [13]. The risk of this situation is failing to include a variety of points of view. This is the so-called incomplete feedback pitfall. To avoid this situation, Helaba creates an open, shared workspace where designers and team members can participate in the argumentation and validation of design decisions in relation to evolving design artefacts. This is illustrated by a domain expert.

"At first I thought [the styles feature] was a whimsical thing [...], but it can be used if you want to achieve certain values on your picture... it's a good way to measure people's emotions when they see something." [Game designer] 


\subsection{Tracking Down Artefact Evolution with Helaba}

Design decisions are communicated to stakeholders, such as project managers or clients, who often do not have design background themselves. Designers frequently struggle to communicate design decisions to these stakeholders, as they might end up expressing rationalizations that have little to do with their actual inspiration [2]. These situations might lead to the multidisciplinary decision-makers pitfall, as designers risk having to dismiss novel ideas in favour of more traditional ones, which are easier to interpret for stakeholders [2]. From a designer point of view, this often leads to suboptimal results.

Helaba allows multidisciplinary teams to annotate artefacts and reflect on the design process and helps to communicate it to relevant stakeholders. As design decisions evolve over time, stakeholders could benefit of being informed about decisions in which they were not involved, or be reminded of past decisions [4]. We envision Helaba could be useful for stakeholders to become aware of the evolution of designs, grasping the efforts invested in the design process, and of other explored but not implemented design choices [11]. This is explained by one of the domain experts.

"[Design processes are] not always friendly in real life, and it's good to keep track of who said what. This is done now by e-mail, but I like [about Helaba] that I can point out [in the artefact]". [Graphical designer \& illustrator]

\section{$6 \quad$ Future Work and Conclusion}

Our research offers insights in how to support communication revolving around design artefacts and activities and provides a set of solutions, as concrete design concepts and strategies. The work presented in this paper has also a set of limitations on which we elaborate further in this section. The Helaba prototype is validated in an early stage with a limited number of domain experts. The scenarios we use to illustrate how Helaba can support design teams only covers a limited number of situations and is not complete.

Despite these limitations, the early validation with domain experts demonstrated three promising directions for extending the prototype. The first direction is to provide richer communication features within the shared space. For instance, by allowing users to add the reasons behind the votes given in the 5-points star-rating scale (see Figure 3 ), which enables a space for critical design and discussion. Second, further investigations should be directed to explore the navigation across different versions of an artefact. This includes extending the history feature to provide teams with a "big picture" of design processes without being overwhelming. The final possibility to extend the prototype is to support the inclusion of different media, e.g. videos, as the current system prototype only considers images.

To conclude, we found the need to create more comprehensive development of communication tools to support design activities. This is reflected in the fact that despite the many different remote communication technologies available today, existing solutions fail to support comprehensive communication on design activities and artefacts. 
Helaba fills this gap by providing a shared workspace for teams to integrate communication with the rationale and evolution of design artefacts over time. We believe this has the potential of leading to more satisfying outcomes from design processes.

\section{Acknowledgments}

This research is supported by the COnCEPT project, funded by the European Commission 7th Framework ICT Research Programme (project no: 610725). We give special thanks to Karel Robert for his involvement in the creation of Helaba, and to the designers involved in our studies.

\section{References}

1. Gutierrez Lopez, M., Haesen, M., Luyten, K., Coninx, K.: Study and analysis of collaborative design practices. In: PIN-C'15, pp. 176-183. (2015)

2. Eckert, C., Stacey, M.: Sources of inspiration: a language of design. Des. Stud. 21, 523-538 (2000)

3. Sharmin, M., Bailey, B.P.: Making sense of communication associated with artifacts during early design activity. In: Campos, P., et al. (eds.) INTERACT 2011. LNCS, vol. 6946, pp. 181-198. Springer, Heidelberg (2011)

4. MacLean, A., Young, R.M., Bellotti, V., Moran, T.: Questions, Options, and Criteria: Elements of Design Space Analysis. Human-Computer Interact. 6, 201-250 (1991)

5. Reeves, B., Shipman, F.: Supporting communication between designers with artifactcentered evolving information spaces. In: CSCW'92, pp. 394-401. (1992)

6. Martinie, C., Palanque, P., Winckler, M., Conversy, S.: DREAMER: A Design Rationale Environment for Argumentation, Modeling and Engineering Requirements. In: SIGDOC'10, pp. 73-80. (2010)

7. Shum, S.B., Hammond, N.: Argumentation-based design rationale: what use at what cost. Int. J. Hum. Comput. Stud. 40, 603-652 (1994)

8. Lee, J.: SIBYL : A Tool for Managing Group Decision Rationale. In: CSCW'90, pp. 79-92. (1990)

9. Schubanz, M.: Design Rationale Capture in Software Architecture: What has to be captured?. In: WCOP'14, pp. 31-36. (2014)

10. Houben, S., Bardram, J.E., Vermeulen J., Luyten, K., Coninx, K.: Activity-Centric Support for Ad Hoc Knowledge Work - A Case Study of co-Activity Manager. In: CHI'13, pp. 22632272. (2013)

11. Fischer, G.: Social creativity: Turning barriers into opportunities for collaborative design. In: PDC'04, pp. 152-161. (2004)

12. Tan, C.S.S., Luyten, K., van den Bergh, J., Schöning, J., Coninx, K.: The Role of Physiological Cues during Remote Collaboration. Presence Teleoperators Virtual Environ. 23, 90-107 (2014)

13. Dourish, P., Bellotti, V.: Awareness and Coordination in Shared Workspaces. In: CSCW'92, pp. 107-114. (1992) 